Gómez, E. J., y González, G. (2015). Comportamiento del aceite de palma de Colombia en los principales mercados de exportación. Revista Lebret, 7. Bucaramanga, Colombia: Universidad Santo Tomás, pp. 283 - 305. ISSN: 2145-5996.

\title{
Comportamiento del aceite de palma de Colombia en los principales mercados de exportación*
}

\section{Colombian Palm Oil Behavior on The Main Export Markets}

Edgar Javier Gómez Parada

Gustavo González Sarmiento

\begin{abstract}
Resumen
El artículo presenta un análisis para determinar la competitividad del aceite de palma de Colombia en los mercados internacionales, mediante variables de participación y comportamiento en los mercados a los que se exportan dichos productos. Se diseña una matriz para analizar el comportamiento en los mercados de exportación entre 2008 - 2012, y se aplican indicadores de balanza comercial relativa y ventajas comparativas. Se evidencia que el aceite de palma, Colombia presenta ventajas en los mercados destino y saldo comercial positivo, relativamente alto en casi todos los mercados, pero dichas exportaciones no son constantes, sino que presentan variaciones y las participaciones son muy bajas.
\end{abstract}

Palabras clave

Análisis competitividad, competitividad aceite de palma, aceite de palma de Colombia.

Códigos de clasificación JEL: F14, L69

\begin{abstract}
The article presents an analysis to determine the competitiveness of Colombian palm oil in international markets through participation and behavior variables in the markets to which such products are exported. A matrix is designed to analyze the behavior in export markets between 2008 - 2012 and relative trade balance indicators and comparative advantages are applied. It is evident that regarding palm oil, Colombia has advantages in relatively high target markets and a positive trade balance in almost all markets, but these exports are not constant, but with variations and stakes are very low.
\end{abstract}

\section{Keywords}

Competitiveness analysis, palm oil competitiveness, Colombian palm oil.

* $\quad$ El artículo muestra apartes del resultado de un proyecto de investigación dentro de la línea de internacionalización de sectores productivos, enfocado a determinar la competitividad del aceite de palma y aceite de almendra de palma de Colombia en el contexto internacional, desarrollado por el grupo de investigación Integración y Globalización de los Negocios de la Facultad de Negocios Internacionales de la Universidad Santo Tomás Seccional Bucaramanga.

1 Magíster en Educación. Docente Facultad de Negocios Internacionales, Universidad Santo Tomás Seccional Bucaramanga. Vinculado al grupo de investigación Integración y Globalización de los Negocios. Correo electrónico: edjagomezp@yahoo.com

2 Especialista en Desarrollo Regional. Docente Facultad de Negocios Internacionales, Universidad Santo Tomás Seccional Bucaramanga. Vinculado al grupo de investigación Integración y Globalización de los Negocios. Correo electrónico: gusta_gsar@hotmail.com 


\section{Introducción}

La dinámica de la competitividad de los sectores productivos del país se ha constituido, en los últimos años, en un tema de preocupación permanente que compromete, no solo a los directivos de las organizaciones empresariales, sino a quienes formulan políticas públicas que tienen como objetivo un mejor posicionamiento del país en el complejo entorno internacional de principios de siglo. Es por ello que se justifica plenamente el ejercicio académico de acercarse a las diferentes perspectivas conceptuales sobre competitividad, pero especialmente, a los métodos propuestos para hacer de ella una categoría mesurable, en condiciones de confiabilidad y practicidad.

La mayoría de los análisis de competitividad de los sectores manufactureros y agroindustriales se han enfocado a evaluar el desempeño del sector en el lugar de origen, y se sustenta en el estudio de la diversidad de los factores que la determinan, relacionados con la eficiencia y la productividad en las variables de producción o las condiciones del entorno que favorecen u obstaculizan la competencia internacional. Son menores los estudios que abordan el tema desde la perspectiva de la dinámica de penetración en diferentes mercados externos de referencia, por lo que el trabajo apunta a desarrollar esta perspectiva complementaria. Se ha seleccionado el sector palmicultor por ser una actividad económica agroindustrial de gran relevancia económica y social para Colombia, que presenta múltiples alternativas de industrialización y de generación de empleo (Olivera, et al., 2011).

Del fruto de la planta de palma africana se extraen dos aceites, uno de la pulpa, que es el aceite de palma propiamente dicho, y otro de la semilla que es el aceite de almendra de palma o palmiste. El presente estudio se enfoca específicamente al aceite de palma con el código 1511 dentro de la clasificación arancelaria. El sector de palma de aceite en Colombia ha logrado un posicionamiento internacional en términos de producción y de exportaciones, tanto que el país se ubica como el cuarto productor mundial y está dentro de los primeros diez países exportadores (2012), presentando un alto dinamismo expresado en términos de áreas cultivadas y producción, por lo que es considerado un sector estratégico dentro de la agroindustria colombiana.

Según datos de Fedepalma (2013) en el 2012 el estimado de hectáreas sembradas en cultivos de palma en Colombia fue de 452 435; con producción de 973703 toneladas de aceite de palma en bruto.

\section{Los conceptos de competitividad y la forma de medirla}

El concepto de competitividad tiene sus orígenes en la teoría económica, específicamente relacionada con el comercio internacional. Las primeras propuestas se atribuyen a David Ricardo, quien introdujo el concepto de ventajas comparativas como elementos diferenciadores basados en factores de producción, que incidían 
directamente en la productividad, como la mano de obra (Labarca, 2007). Las ventajas comparativas muestran el desempeño en el mercado internacional a través del tiempo y se pueden determinar para distintos sectores o actividades económicas. Dentro de este concepto, las empresas de una región exportan bienes para los cuales han logrado una buena utilización de los factores disponibles, es decir, crear unas ventajas sobre sus competidores. A partir de ello, aquellos sectores y empresas que logran permanecer en los mercados internacionales son más competitivos que otros que no logran hacerlo. Un país tiene unos sectores que son más competitivos que otros, tanto en el mercado interno como en el mercado internacional. De ahí que las empresas para poder ingresar y posicionarse en mercados externos, tengan que construir ventajas sobre los demás competidores (Jung, Garbarino, Jerusalmi, Durán, y Plottier, 2006).

El modelo de Heckscher - Ohlin agrega un nuevo factor, el capital, y propone que la manera como se combina el capital y el trabajo genera diversas formas de producción, que son las que establecen diferencias de producción y de comercialización y son las bases para competir, de acuerdo con Artal, Castillo y Requena (2006). A partir de estos postulados, se avanzó con varios trabajos que enriquecieron las teorías y conceptos relacionados con competitividad; enfocados a explicar cómo algunos países, empresas y productos logran posicionarse con mayor éxito en los mercados y obtener mayores dividendos que sus competidores. En las últimas décadas del siglo pasado, como respuesta al cambio del modelo de desarrollo y a las tendencias crecientes de interrelación entre las economías del mundo, el debate alrededor de los determinantes de la competitividad empresarial y regional y a las posibilidades de medirla, se han enriquecido con los aportes de múltiples estudiosos, quienes, desde diferentes orillas, han enriquecido la perspectiva teórica y los estudios empíricos.

Entre los diversos aportes cabe destacar el postulado de ciclo de vida del producto, propuesto por Raymond Vernon, señalando que los productos pasan por diversas etapas sucesivas: introducción, crecimiento, madurez y declive y responden a necesidades y oportunidades del mercado por lo que se hace necesario estar en permanente innovación de productos (Villazul, 2004).

Se aprecia que la competitividad tiene relación directa con el aprovechamiento de recursos que se ponen a disposición de la producción, para lograr menores costos que los competidores, buena calidad y diferenciación. De allí que el concepto de competitividad ha venido evolucionando hasta llegar a nuevos postulados, como el de ventajas competitivas soportadas en la capacidad de transformar insumos para producir bienes de una manera diferencial frente a los competidores, logrando la maximización de utilidades. Esta nueva visión multicasual y compleja ha relegado a un segundo plano el concepto de ventajas comparativas de Ricardo, en la medida que ya no son las condiciones geográficas o la presencia de recursos naturales los únicos condicionantes de la posición relativa de los países en los mercados internacionales (Lombana y Rosas, 2009). 
Para Michael Porter (1999), uno de los más reconocidos estudiosos del tema, la competitividad es un atributo o cualidad de las empresas, no de los países. La competitividad empresarial está asociada con cuatro fuerzas: condiciones de los factores, condiciones de la demanda, industrias conexas y de apoyo, y estructura y rivalidad entre las empresas. La interacción entre estas fuerzas representa la estrategia empresarial para competir. De ahí que una de las tendencias para los análisis de competitividad es el llamado diamante de competitividad de Porter, en el que se evalúa cada una de las fuerzas centradas específicamente en la región en la que está ubicada la empresa. El concepto de competitividad empresarial en la perspectiva Porter y Kramer (2006) remite, entonces, a la capacidad y las estrategias que adopta la unidad productiva para producir y ubicar sus productos en el mercado internacional dentro de un ambiente donde existen otras empresas de otros países. Es decir, la empresa debe desarrollar unas capacidades propias y diferenciales, para que pueda desenvolverse con éxito en mercados nacionales e internacionales, según (Benítez, 2007). En ese orden de ideas, los indicadores de medición de la competitividad se estructuran partiendo de los factores que inciden en la productividad y eficiencia de la empresa a nivel interno y de las variables del entorno que condicionan su comportamiento y evolución.

Sharples y Milhan (1990), citados por Chavarría, Rojas y Sepúlveda (2002), definen la competitividad en función de los mercados externos a los cuales se lleva un producto, por lo cual se espera obtener un beneficio. En esos términos, la competividad de una empresa o industria se interpreta como la habilidad de ubicar bienes y servicios en el tiempo, lugar y forma requeridos por los clientes extranjeros a precios iguales o mejores de los de otros competidores potenciales, y por ello se obtienen retornos adecuados a los recursos empleados. Desde esa perspectiva que asumimos como referente en este estudio, la competitividad se entiende como el desempeño de una empresa o sector en un mercado donde se enfrenta a otros competidores.

Son varios los esfuerzos que se han hecho para estructurar indicadores que permitan medir la competitividad utilizando el criterio de penetración en los mercados. Hace algunos años, por ejemplo, Bonifaz y Mortimore (1999, p. 26) desde la CEPAL propusieron la metodología CAN que, según sus propias palabras: "se basa en la evaluación del nivel de penetración y participación de los países en un ámbito de comercio específico, sin buscar hacer referencia a los factores explicativos".

Dentro de esta visión es interesante destacar la perspectiva según la cual, los patrones de comercio de un país, terminan por "revelar" su posición competitiva. Balassa hizo notar que, como resultado de la multiplicidad de los factores determinantes de la competitividad y de la dificultad para cuantificar algunos de ellos, en vez de plantear principios generales y tratar de aplicarlos a la realidad podía ser más efectivo el análisis del comportamiento de los patrones actuales de comercio 
de los países. Así, propuso el Índice de Ventaja Comparativa Revelada (IVCR) ${ }^{3}$ (de Pablo \& Battistuzzi, 2012).

\section{Aspectos metodológicos}

Para analizar el comportamiento del comercio internacional de Colombia en productos de aceite de palma, se seleccionan los ocho principales mercados destino de las exportaciones: Holanda, Brasil, México, Chile, Estados Unidos, República Dominicana, Alemania y Panamá. Se toman como variables:

- Participación de Colombia en el mercado destino: relación entre las importaciones totales del mercado destino y las importaciones provenientes de Colombia.

- Participación del mercado destino en las exportaciones de Colombia: relación entre el total exportado por Colombia y lo que exporta específicamente al mercado destino.

- Principales proveedores al mercado destino en aceite de palma: se toma la participación de los principales países exportadores al mercado destino en términos porcentuales en el 2012 y la variación en el mercado entre 2008 2012.

- Importaciones de otros aceites vegetales realizadas por el mercado destino: se toma la participación en términos porcentuales de las importaciones realizadas por el mercado en el 2012 en aceites vegetales.

- Arancel aplicado por el mercado a los países exportadores: se toma el valor porcentual que aplica el mercado para el ingreso del producto al país exportador en el 2012.

Posteriormente, se aplican indicadores de balanza comercial relativa, balanza comercial relativa con referencia a cada país destino e Índice de ventaja comparativa revelada en cada mercado.

El periodo de análisis es de cinco años (2008 - 2012). Los datos se toman directamente de la base de datos TRADEMAP ${ }^{4}$, para la partida arancelaria 1511: Aceite de palma y sus fracciones, incluso refinado, pero sin modificar.

3 El índice VCR puede ser definido como un cociente donde el numerador representa la participación del bien "i" en las exportaciones totales del país y el denominador indica la participación de las exportaciones mundiales del bien "i" en las exportaciones mundiales totales. En suma, se compara la estructura de las exportaciones de un determinado país con la estructura de las ventas externas mundiales. La convención usual es que si el índice es mayor que uno, el país en cuestión "revela" una ventaja comparativa en dicho bien o sector y si es menor que uno "revela" una desventaja comparativa.

4 Base de datos del International Trade Centre que suministra estadísticas sobre datos comerciales mensuales, trimestrales y anuales. Valores de importación y exportación, volúmenes, tasas de crecimiento, cuotas de mercado, etc. 


\section{Comercio exterior de aceite de palma de Colombia}

El aceite de palma es el de mayor producción mundial entre los aceites oleaginosos y el de más exportación; Indonesia y Malasia son los mayores productores y exportadores. Colombia ocupa (en el 2012) el cuarto lugar en producción y el 9 lugar entre los exportadores.

Tabla 1. Exportaciones de Colombia de aceite de palma (1511) Valores en miles de dólares

\begin{tabular}{|c|c|c|c|c|c|c|c|}
\hline Destino de las exp. & 2008 & 2009 & 2010 & 2011 & 2012 & $\begin{array}{c}\text { Variación } \\
2008-2012\end{array}$ & $\begin{array}{c}\text { Participación } \\
2012\end{array}$ \\
\hline País & 320344 & 138624 & 83180 & 191059 & 188985 & $-41,01$ & \\
\hline Holanda & 25272 & 8139 & 6342 & 75561 & 71331 & 182,25 & 37,74 \\
\hline Brasil & 28914 & 13984 & 3352 & 45217 & 34133 & 18,05 & 18,06 \\
\hline México & 1556 & 31696 & 25547 & 5047 & 31182 & 1903,98 & 16,50 \\
\hline Chile & 5385 & 3999 & 6021 & 7542 & 7886 & 46,44 & 4,17 \\
\hline Panamá & 4880 & 3205 & 2954 & 5988 & 6912 & 41,64 & 3,66 \\
\hline Estados Unidos & 14493 & 3582 & 4355 & 4705 & 4227 & $-70,83$ & 2,24 \\
\hline $\begin{array}{l}\text { República } \\
\text { Dominicana }\end{array}$ & 8504 & 8386 & 6835 & 11851 & 16951 & 99,33 & 8,97 \\
\hline Alemania & 131273 & 38200 & 295 & 14161 & 3135 & $-97,61$ & 1,66 \\
\hline Trinidad y Tobago & 5602 & 2588 & 1714 & 2440 & 1982 & $-64,62$ & 1,05 \\
\hline Australia & 76 & 97 & 280 & 708 & 1319 & 1635,53 & 0,70 \\
\hline Bélgica & 1483 & 1943 & 3502 & 3252 & 1568 & 5,73 & 0,83 \\
\hline Canadá & 0 & 59 & 44 & 125 & 679 & & 0,36 \\
\hline Japón & 165 & 217 & 398 & 514 & 372 & 125,45 & 0,20 \\
\hline Guyana & 472 & 341 & 231 & 628 & 414 & $-12,29$ & 0,22 \\
\hline Perú & 1226 & 24 & 842 & 0 & 192 & $-84,34$ & 0,10 \\
\hline Reino Unido & 54726 & 17061 & 8937 & 8613 & 1352 & $-97,53$ & 0,72 \\
\hline España & 1446 & 62 & 0 & 15 & 2156 & 49,10 & 1,14 \\
\hline Venezuela & 27835 & 2472 & 9833 & 976 & 960 & $-96,55$ & 0,51 \\
\hline
\end{tabular}

Fuente: TRADE MAP. Elaboración propia.

Las exportaciones de aceite de palma de Colombia disminuyeron $41 \%$ entre 2008 - 2012 decrecimiento que se refleja principalmente en los mercados de Estados Unidos y de Alemania; los principales destinos de las exportaciones en el 2012 fueron Holanda, Brasil y México, mercados que participan con 72,3\% y 
presentaron aumentos en el periodo analizado. Se exporta a otros mercados con bajas participaciones.

Tabla 2. Importaciones de Colombia de aceite de palma (1511)

Valores en miles de dólares

\begin{tabular}{|l|c|c|c|c|c|c|c|}
\hline $\begin{array}{c}\text { Origen de las } \\
\text { import. }\end{array}$ & $\mathbf{2 0 0 8}$ & $\mathbf{2 0 0 9}$ & $\mathbf{2 0 1 0}$ & $\mathbf{2 0 1 1}$ & $\mathbf{2 0 1 2}$ & $\begin{array}{c}\text { Variación } \\
\mathbf{2 0 0 8}-\mathbf{2 0 1 2}\end{array}$ & $\begin{array}{c}\text { Participación } \\
\mathbf{2 0 1 2}\end{array}$ \\
\hline Mundo & 27523 & 51442 & 105680 & 139557 & 138469 & 403,1 & \\
\hline Ecuador & 27147 & 50319 & 40895 & 45620 & 74576 & 174,7 & 53,86 \\
\hline Indonesia & 0 & 0 & 2555 & 17135 & 41238 & & 29,78 \\
\hline Malasia & 341 & 1029 & 23466 & 41500 & 11593 & 3299,7 & 8,37 \\
\hline Brasil & 0 & 0 & 5454 & 3 & 7 & & 0,01 \\
\hline
\end{tabular}

Fuente: TRADE MAP. Elaboración propia.

Colombia ha aumentado en $403 \%$ las exportaciones de aceite de palma entre 2008 - 2012, comparado con la disminución de las exportaciones en el mismo periodo, se refleja una baja en la tendencia exportadora del producto. Sin embargo, la balanza comercial del producto ha sido positiva para Colombia en casi todos los años, a excepción del 2010 y presentó una disminución de 82,7\% entre 2008 - 2012.

La balanza comercial para Colombia del aceite de palma en bruto ha presentado fluctuaciones entre 2008 - 2012, con picos altos en el 2008, cuando se presenta el mayor superávit y picos bajos en el 2010 cuando se presenta déficit. En el 2011 y 2012 vuelve a recuperar a favor de las exportaciones. Se infiere que se presentan amenazas por importaciones del producto que puede desplazar la producción nacional. Es importante señalar que la demanda interna del producto ha aumentado debido a la producción de biodiesel en Colombia.

Tabla 3. Balanza comercial de Colombia de aceite de palma (1511)

Valores en miles de dólares

\begin{tabular}{|c|c|c|c|c|c|}
\hline $\mathbf{2 0 0 8}$ & $\mathbf{2 0 0 9}$ & $\mathbf{2 0 1 0}$ & $\mathbf{2 0 1 1}$ & $\mathbf{2 0 1 2}$ & Variación 2008-2012 \\
\hline 292821 & 87182 & -22500 & 51502 & 50516 & $-82,7$ \\
\hline
\end{tabular}

Fuente: TRADE MAP. Elaboración propia.

\section{Desempeño de Colombia en los mercados a los cuales exporta aceite de palma}

Para analizar el desempeño de Colombia en cada mercado de exportación, se determina la participación de Colombia en el mercado destino, mediante una relación entre las exportaciones de Colombia (importaciones del mercado destino que realiza desde Colombia) y las importaciones totales que hace el mercado destino (provenientes de todo el mundo). También se establece una relación entre las exportaciones totales 
de Colombia y las exportaciones al mercado destino. Se identifica la participación de otros aceites oleaginosos en el mercado importador y el arancel que se aplica a los participantes en el mercado. En un cuadro matriz se presentan las variables. Se hace el ejercicio para los principales ocho mercados destino de las exportaciones de Colombia en aceite de palma, Holanda, Brasil, México, Chile, Estados Unidos, República Dominicana, Panamá y Alemania.

\section{Mercado de Holanda}

Holanda en el 2012 realizó importaciones de aceite de palma por más de 2,7 mil millones de dólares y tuvo aumento del 44,\% entre 2008 - 2012, por lo que se considera un mercado creciente y atractivo para los productores y exportadores de este producto.

El comportamiento de Colombia en el mercado holandés es favorable, por cuanto presenta un crecimiento superior al comportamiento del mercado y al de los dos principales competidores entre 2008 - 2012, también aumentó la participación en el mercado de 1,32\% en el 2008, a 2,59\% en el 2012. En el 2011 y 2012 el mercado de Holanda es el principal destino de las exportaciones de aceite de palma de Colombia.

Los principales competidores en el mercado de Holanda son Indonesia, Malasia y Nueva Guinea, los dos primeros con alta participación. Colombia tiene la ventaja de menor arancel para el ingreso del producto frente a los dos principales participantes en el mercado.

En el mercado holandés el aceite de palma es el de mayor participación en las importaciones de aceites oleaginosos, con 55,8\%. Seguido por el aceite de girasol y aceite de colza. El aceite de soya tiene poca participación y presenta disminución entre 2008 - 2012, por lo que no se ve amenaza de desplazamiento de productos sustitutos para el aceite de palma en el mediano plazo en dicho mercado.

Tabla 4. Desempeño de Colombia en exportaciones de aceite de palma (1511) en el mercado de Holanda - Valores en miles de dólares

\begin{tabular}{|l|c|c|c|c|c|c|}
\cline { 2 - 7 } \multicolumn{1}{l|}{} & $\mathbf{2 0 0 8}$ & $\mathbf{2 0 0 9}$ & $\mathbf{2 0 1 0}$ & $\mathbf{2 0 1 1}$ & $\mathbf{2 0 1 2}$ & Variación 2009 - 2013 \\
\hline $\begin{array}{l}\text { Colombia exporta hacia } \\
\text { el mundo }\end{array}$ & 320344 & 138624 & 83180 & 191059 & 188985 & $-41,01$ \\
\hline $\begin{array}{l}\text { Holanda importa de } \\
\text { Colombia }\end{array}$ & 26443 & 7850 & 6342 & 60049 & 59211 & 123,92 \\
\hline $\begin{array}{l}\text { Holanda importa del } \\
\text { mundo }\end{array}$ & 1913695 & 1312482 & 1448959 & 1836388 & 2749623 & 43,68 \\
\hline $\begin{array}{l}\text { Participación de } \\
\text { Colombia en el } \\
\text { mercado destino }\end{array}$ & $1,38 \%$ & $0,60 \%$ & $0,44 \%$ & $3,27 \%$ & $2,15 \%$ & $55,84 \%$ \\
\hline $\begin{array}{l}\text { Participación del } \\
\text { mercado destino en } \\
\text { las exportaciones de } \\
\text { Colombia }\end{array}$ & $8,25 \%$ & $5,66 \%$ & $7,62 \%$ & $31,43 \%$ & $31,33 \%$ & $279,56 \%$ \\
\hline
\end{tabular}




\begin{tabular}{|c|c|c|c|}
\hline \multirow{5}{*}{$\begin{array}{l}\text { Principales } \\
\text { proveedores al mercado } \\
\text { de Holanda en aceite } \\
\text { de palma } \\
\text { (2012) }\end{array}$} & $\begin{array}{l}\text { Principales proveedores en el } \\
\text { mercado (2012) }\end{array}$ & $\begin{array}{l}\text { Participación en el } \\
2012\end{array}$ & Variación 2008 - 2012 \\
\hline & Malasia & $44,14 \%$ & $20,41 \%$ \\
\hline & Indonesia & $36,53 \%$ & $53,40 \%$ \\
\hline & Nueva Guinea & $5,35 \%$ & $132,89 \%$ \\
\hline & Honduras & $3,64 \%$ & $827,59 \%$ \\
\hline \multirow{6}{*}{$\begin{array}{l}\text { Importaciones de } \\
\text { aceites vegetales en } \\
\text { el } 2012\end{array}$} & Aceites vegetales & $\begin{array}{l}\text { Participación en el } \\
2012\end{array}$ & Variación 2008 - 2012 \\
\hline & Aceite de palma & $55,8 \%$ & $43,68 \%$ \\
\hline & Aceite de girasol & $13,29 \%$ & $3,51 \%$ \\
\hline & Aceite de colza & $15,31 \%$ & $56,42 \%$ \\
\hline & Aceite de almendra de palma & $12,92 \%$ & $13,43 \%$ \\
\hline & Aceite de soya & $3,04 \%$ & $-39,16 \%$ \\
\hline $\begin{array}{l}\text { Arancel aplicado por } \\
\text { Holanda para el ingreso } \\
\text { de producto } \\
\text { Año } 2012\end{array}$ & \multicolumn{3}{|l|}{$\begin{array}{l}\text { Indonesia: } 5,8 \% \\
\text { Malasia: } 5,8 \% \\
\text { Nueva Guinea: } 0 \% \\
\text { Colombia: } 0 \%\end{array}$} \\
\hline
\end{tabular}

Fuente: Elaboración propia.

\section{Mercado de Brasil}

Brasil realizó importaciones por 230 mil millones de dólares en aceite de palma en el 2012 y presentó crecimiento de $34 \%$ entre 2008 - 2012, por tanto es un mercado dinámico y altamente atractivo para los países productores y exportadores.

El comportamiento de Colombia en el mercado entre 2008 - 2012 fue decreciente $(-26,64 \%)$ y disminuyó la participación en el mismo periodo $(-45,29 \%)$, por lo que se evidencia pérdida para el producto en aceite de palma en dicho mercado. Aún así el mercado de Brasil es altamente significativo para Colombia en aceite de palma, por el volumen de exportaciones alcanzado (más de 34 millones de dólares en el 2012) y porque es el segundo país proveedor despúes de Indonesia.

Ecuador es un nuevo competidor en el mercado de Brasil y se puede considerar competidor muy fuerte para Colombia por la similitud de ventajas en el mercado en términos de distancia geográfica y aranceles. Indonesia es el principal participante en el mercado con más del 63\% y el único con crecimiento entre 2008 - 2012 respecto a los primeros competidores.

En el mercado de Brasil el aceite de oliva tiene mayor participación en las importaciones sobre los demás aceites oleaginosos con crecimiento similar a las importaciones de aceite de palma entre 2008 - 2012; menor participación tienen el aceite de girasol (aunque con mayor crecimiento) y el aceite de colza. 
Tabla 5. Desempeño de Colombia en exportaciones de aceite de palma (1511) en el mercado de Brasil - Valores en miles de dólares

\begin{tabular}{|c|c|c|c|c|c|c|}
\hline & 2008 & 2009 & 2010 & 2011 & 2012 & Variación 2009 - 2013 \\
\hline $\begin{array}{l}\text { Colombia exporta hacia } \\
\text { el mundo }\end{array}$ & 320344 & 138624 & 83180 & 191059 & 188985 & $-41,01$ \\
\hline $\begin{array}{l}\text { Brasil importa de } \\
\text { Colombia }\end{array}$ & 46475 & 8308 & 9061 & 45289 & 34093 & $-26,64$ \\
\hline $\begin{array}{l}\text { Brasil importa del } \\
\text { mundo }\end{array}$ & 171939 & 91883 & 124816 & 246180 & 230531 & 34,08 \\
\hline $\begin{array}{l}\text { Participación de } \\
\text { Colombia en el } \\
\text { mercado destino }\end{array}$ & $27,03 \%$ & $9,04 \%$ & $7,26 \%$ & $18,40 \%$ & $14,79 \%$ & $-45,29 \%$ \\
\hline $\begin{array}{l}\text { Participación del } \\
\text { mercado destino en } \\
\text { las exportaciones de } \\
\text { Colombia }\end{array}$ & $14,51 \%$ & $5,99 \%$ & $10,89 \%$ & $23,70 \%$ & $18,04 \%$ & $24,35 \%$ \\
\hline \multirow{5}{*}{$\begin{array}{l}\text { Principales } \\
\text { proveedores al mercado } \\
\text { de Brasil en aceite de } \\
\text { palma (2012) }\end{array}$} & \multicolumn{3}{|c|}{$\begin{array}{l}\text { Principales proveedores en el } \\
\text { mercado (2012) }\end{array}$} & \multicolumn{2}{|c|}{$\begin{array}{l}\text { Participación en el } \\
2012\end{array}$} & Variación 2008 - 2012 \\
\hline & \multicolumn{3}{|l|}{ Indonesia } & \multicolumn{2}{|c|}{$63,38 \%$} & $45,53 \%$ \\
\hline & \multicolumn{3}{|l|}{ Colombia } & \multicolumn{2}{|c|}{$14,79 \%$} & $-26,64 \%$ \\
\hline & \multicolumn{3}{|l|}{ Ecuador } & \multicolumn{2}{|c|}{$12,00 \%$} & - \\
\hline & \multicolumn{3}{|l|}{ Malasia } & \multicolumn{2}{|c|}{$2,98 \%$} & $-68,83 \%$ \\
\hline \multirow{7}{*}{$\begin{array}{l}\text { Importaciones de } \\
\text { aceites vegetales en el } \\
\text { año } 2012\end{array}$} & \multicolumn{3}{|c|}{ Aceites vegetales } & \multicolumn{2}{|c|}{$\begin{array}{l}\text { Participación en el } \\
2012\end{array}$} & Variación 2008 - 2012 \\
\hline & \multicolumn{3}{|c|}{ Aceite de oliva } & \multicolumn{2}{|c|}{$38,35 \%$} & $34,83 \%$ \\
\hline & \multicolumn{3}{|c|}{ Aceite de almendra de palma } & \multicolumn{2}{|c|}{$25,99 \%$} & $68,05 \%$ \\
\hline & \multicolumn{3}{|c|}{ Aceite de palma } & \multicolumn{2}{|c|}{$28,36 \%$} & $34,08 \%$ \\
\hline & \multicolumn{3}{|c|}{ Aceite de girasol } & \multicolumn{2}{|c|}{$5,95 \%$} & $76,19 \%$ \\
\hline & \multicolumn{3}{|c|}{ Aceite de colza } & \multicolumn{2}{|c|}{$1,20 \%$} & $-56,65 \%$ \\
\hline & \multicolumn{3}{|c|}{ Aceite de soya } & \multicolumn{2}{|c|}{$0,15 \%$} & $-95,92 \%$ \\
\hline $\begin{array}{l}\text { Arancel aplicado por } \\
\text { Brasil para el ingreso } \\
\text { de producto }\end{array}$ & \multicolumn{6}{|c|}{$\begin{array}{l}\text { Indonesia } 10 \% \\
\text { Colombia: } 0 \\
\text { Ecuador: } 0 \\
\text { Malasia: } 10 \%\end{array}$} \\
\hline
\end{tabular}

Fuente: Elaboración propia.

\section{Mercado de México}

El mercado de México realizó en el 2012 importaciones en aceite de palma por valor aproximado de 476 mil millones de dólares y presentó crecimiento de 36,65\% entre 2008 - 2012, por tanto es un mercado dinámico y atractivo para los productores y exportadores.

Colombia aumentó significativamente las exportaciones en aceite de palma al mercado de México entre 2008 - 2012 en 1622\% y superó los 29 millones de dólares en el 2012 y alcazó una participación de 6,16\% en dicho año. El crecimiento 
de Colombia estuvo por encima de los principales competidores, lo que evidencia ganancia en el mercado para este producto.

Los principales participantes en el mercado de México son Guatemala, Costa Rica y Honduras con mayor valor en las exportaciones que Colombia en el 2012, pero con crecimientos inferiones entre 2008 - 2012. Se destaca que los principales copartícipes en el mercado son geográficamente cercanos a México, los tres primeros son países centroamericanos y todos pagan el mismo arancel.

El aceite de palma es el principal aceite oleaginoso que importa México por encima del aceite de soya y de copra. No se evidencia amenaza de sustitución del producto por otro aceite en corto plazo.

Tabla 6. Desempeño de Colombia en exportaciones de aceite de palma (1511) en el mercado de México - Valores en miles de dólares

\begin{tabular}{|c|c|c|c|c|c|c|}
\hline & 2008 & 2009 & 2010 & 2011 & 2012 & Variación 2009 - 2013 \\
\hline $\begin{array}{l}\text { Colombia exporta } \\
\text { hacia el mundo }\end{array}$ & 320344 & 138624 & 83180 & 191059 & 188985 & $-41,01 \%$ \\
\hline $\begin{array}{l}\text { México importa de } \\
\text { Colombia }\end{array}$ & 1703 & 35956 & 24158 & 3846 & 29329 & $1622,20 \%$ \\
\hline $\begin{array}{l}\text { México importa del } \\
\text { mundo }\end{array}$ & 348512 & 265977 & 314303 & 435868 & 476253 & $36,65 \%$ \\
\hline $\begin{array}{l}\text { Participación de } \\
\text { Colombia en el } \\
\text { mercado destino }\end{array}$ & $0,49 \%$ & $13,52 \%$ & $7,69 \%$ & $0,88 \%$ & $6,16 \%$ & $1160,27 \%$ \\
\hline $\begin{array}{l}\text { Participación del } \\
\text { mercado destino en } \\
\text { las exportaciones de } \\
\text { Colombia }\end{array}$ & $0,53 \%$ & $25,94 \%$ & $29,04 \%$ & $2,01 \%$ & $15,52 \%$ & $2819,25 \%$ \\
\hline \multirow{5}{*}{$\begin{array}{l}\text { Principales } \\
\text { proveedores de aceite } \\
\text { de palma al mercado de } \\
\text { México (2012) }\end{array}$} & \multicolumn{3}{|c|}{$\begin{array}{c}\text { Principales proveedores en el } \\
\text { mercado (2012) }\end{array}$} & \multicolumn{2}{|c|}{$\begin{array}{c}\text { Participación en el } \\
2012\end{array}$} & Variación 2008 - 2012 \\
\hline & \multicolumn{3}{|c|}{ Guatemala } & \multicolumn{2}{|c|}{$38,19 \%$} & $102,47 \%$ \\
\hline & \multicolumn{3}{|c|}{ Costa Rica } & \multicolumn{2}{|c|}{$31,15 \%$} & $19,43 \%$ \\
\hline & \multicolumn{3}{|l|}{ Honduras } & \multicolumn{2}{|c|}{$13,21 \%$} & $-30,35 \%$ \\
\hline & \multicolumn{3}{|l|}{ Colombia } & \multicolumn{2}{|c|}{$6,16 \%$} & $1622,20 \%$ \\
\hline \multirow{7}{*}{$\begin{array}{l}\text { Importaciones de } \\
\text { aceites vegetales en } \\
\text { el } 2012\end{array}$} & \multicolumn{3}{|c|}{ Aceites vegetales } & \multicolumn{2}{|c|}{$\begin{array}{c}\text { Participación en el } \\
2012\end{array}$} & Variación 2008 - 2012 \\
\hline & \multicolumn{3}{|c|}{ Aceite de palma } & \multicolumn{2}{|c|}{$48.09 \%$} & $36,65 \%$ \\
\hline & \multicolumn{3}{|c|}{ Aceite de soya } & \multicolumn{2}{|c|}{$21,87 \%$} & $-22,49 \%$ \\
\hline & \multicolumn{3}{|c|}{ Aceite de copra } & \multicolumn{2}{|c|}{$9,80 \%$} & $55,48 \%$ \\
\hline & \multicolumn{3}{|c|}{ Aceite de oliva } & \multicolumn{2}{|c|}{$4,73 \%$} & $-9,21 \%$ \\
\hline & \multicolumn{3}{|c|}{ Aceite de colza } & \multicolumn{2}{|c|}{$7,45 \%$} & $10,42 \%$ \\
\hline & \multicolumn{3}{|c|}{ Aceite de girasol } & \multicolumn{2}{|c|}{$8,05 \%$} & $31,46 \%$ \\
\hline $\begin{array}{l}\text { Arancel aplicado por } \\
\text { México para el ingreso } \\
\text { de producto }\end{array}$ & \multicolumn{6}{|c|}{$\begin{array}{l}\text { Guatemala: }(10,3 \%) \\
\text { Costa Rica: }(10,3 \%) \\
\text { Honduras: }(10,3 \%) \\
\text { Colombia: }(10,3 \%)\end{array}$} \\
\hline
\end{tabular}

Fuente: Elaboración propia. 


\section{Mercado de Chile}

Chile es un mercado con un volumen de importación relativamente bajo en aceite de palma y con disminución entre el 2008 - 2012. Por tanto es un mercado con un atractivo medio para los países productores y exportadores. Pero tiene alta significancia para Colombia porque es el principal participante en dicho mercado, con $72,33 \%$ en el 2012, aunque tuvo disminución entre 2008 - 2012 en el valor exportado y en participación.

Otros partícipes en el mercado son Malasia y Ecuador que también presentaron disminución en las exportaciones entre 2008 - 2012. Colombia está libre de arancel para aceite de palma en el mercado chileno, mientras que Malasia debe asumir un arancel del $6 \%$.

Dentro de las importaciones de aceites oleaginosos en el mercado de Chile, el aceite de palma tiene muy baja participación (1,41\% en el 2012), siendo los principales el aceite de colza, el de girasol y el de soya, que además presentaron altos crecimentos en el valor importado entre 2008 - 2012. Por tanto se evidencia desplazamiento y pérdida en el mercado chileno para el aceite de palma por los demás aceites oleaginosos mencionados.

Tabla 7. Desempeño de Colombia en exportaciones de aceite de palma (1511) en el mercado de Chile - Valores en miles de dólares

\begin{tabular}{|c|c|c|c|c|c|c|}
\hline & & & & & & \\
\hline & 2008 & 2009 & 2010 & 2011 & 2012 & Variación 2009 - 2013 \\
\hline $\begin{array}{l}\text { Colombia exporta hacia } \\
\text { el mundo }\end{array}$ & 320344 & 138624 & 83180 & 191059 & 188985 & $-41,01$ \\
\hline $\begin{array}{l}\text { Chile importa de } \\
\text { Colombia }\end{array}$ & 2906 & 894 & 1217 & 1480 & 1349 & $-53,58$ \\
\hline $\begin{array}{l}\text { Chile importa del } \\
\text { mundo }\end{array}$ & 3645 & 975 & 1535 & 2397 & 1865 & $-48,83$ \\
\hline $\begin{array}{l}\text { Participación de } \\
\text { Colombia en el } \\
\text { mercado destino }\end{array}$ & 79,73 & 91,69 & 79,28 & 61,74 & 72,33 & $-9,28$ \\
\hline $\begin{array}{l}\text { Participación del } \\
\text { mercado destino en } \\
\text { las exportaciones de } \\
\text { Colombia }\end{array}$ & 1,68 & 2,88 & 7,24 & 3,95 & 4,17 & 148,21 \\
\hline \multirow{4}{*}{$\begin{array}{l}\text { Principales } \\
\text { proveedores al mercado } \\
\text { de Chile (2012) }\end{array}$} & \multicolumn{3}{|c|}{$\begin{array}{c}\text { Principales proveedores en el } \\
\text { mercado (2012) }\end{array}$} & \multicolumn{2}{|c|}{$\begin{array}{l}\text { Participación en el } \\
2012\end{array}$} & Variación 2008 - 2012 \\
\hline & \multicolumn{3}{|l|}{ Colombia } & \multicolumn{2}{|c|}{72,33} & $-53,58$ \\
\hline & \multicolumn{3}{|l|}{ Malasia } & \multicolumn{2}{|c|}{16,14} & $-37,29$ \\
\hline & \multicolumn{3}{|l|}{ Ecuador } & \multicolumn{2}{|c|}{7,61} & $-45,17$ \\
\hline
\end{tabular}




\begin{tabular}{|l|l|c|c|}
\hline \multirow{4}{*}{$\begin{array}{l}\text { Importaciones de } \\
\text { aceites vegetales en } \\
\text { el } 2012\end{array}$} & $\begin{array}{c}\text { Aceites vegetales } \\
\text { 2012 }\end{array}$ & $19,30 \%$ & Variación 2008 - 2012 \\
\cline { 2 - 4 } & Aceite de soya & $44,87 \%$ & $466,15 \%$ \\
\cline { 2 - 4 } & Aceite de colza & $30,39 \%$ & $306,81 \%$ \\
\cline { 2 - 4 } & Aceite de girasol & $1,41 \%$ & $260,25 \%$ \\
\cline { 2 - 4 } & Aceite de palma & $1,89 \%$ & $-48,43 \%$ \\
\cline { 2 - 4 } & Aceite de almendra de palma & $2,15 \%$ & $-40,08 \%$ \\
\cline { 2 - 4 } & Aceite de oliva & & \\
\hline $\begin{array}{l}\text { Arancel aplicado por } \\
\text { Chile para el ingreso de } \\
\text { producto }\end{array}$ & $\begin{array}{l}\text { Colombia: }(0 \%) \\
\text { Malasia: }(6 \%)\end{array}$ & $\begin{array}{l}\mid \\
\text { Ecuador: }(0,3 \%)\end{array}$ & \\
\hline
\end{tabular}

Fuente: Elaboración propia.

\section{Mercado de República Dominicana}

República Dominicana es un mercado que presentó alto crecimiento entre 2008 - $2012(262,42 \%)$ en aceite de palma con un tamaño relativamente mediano como mercado importador del producto representado en 31 millones de dólares en el 2012.

Colombia también ha aumentado las exportaciones a ese país a un ritmo menor que el desempeño de dicho mercado, eso ha llevado a disminuir la participación, de $97,91 \%$ en 2008 a 52\% en 2012. Esta pérdida de participación se puede sustentar en la aparición de otros países centroamericanos en ese mercado como Honduras y Costa Rica, además de Ecuador.

El atractivo del mercado de República Dominicana se fundamenta en el alto crecimiento y en la alta particpación de Colombia. Es un mercado con alta competencia frente a competidores similares para Colombia, que además debe pagar alto arancel para el ingreso del producto a ese país.

En República Dominicana el aceite de soya es el principal producto importado dentro de los aceites oleaginosos con participación del 69\% en el 2012. El aceite de palma es el segundo importado en el mercado con crecimiento superior a las importaciones de aceite de soya entre 2008 - 2012, lo que indica que el producto ha logrado mayor presencia en el mercado.

Tabla 8. Desempeño de Colombia en exportaciones de aceite de palma (1511) en el mercado de República Dominicana - Valores en miles de dólares

\begin{tabular}{|l|c|c|c|c|c|c|}
\cline { 2 - 6 } \multicolumn{1}{l|}{} & $\mathbf{2 0 0 8}$ & $\mathbf{2 0 0 9}$ & $\mathbf{2 0 1 0}$ & $\mathbf{2 0 1 1}$ & $\mathbf{2 0 1 2}$ & Variación 2009-2013 \\
\hline $\begin{array}{l}\text { Colombia exporta hacia } \\
\text { el mundo }\end{array}$ & 320344 & 138624 & 83180 & 191059 & 188985 & $-41,01$ \\
\hline $\begin{array}{l}\text { República Dominicana } \\
\text { importa de Colombia }\end{array}$ & 8381 & 8386 & 8300 & 15586 & 16133 & 92,49 \\
\hline $\begin{array}{l}\text { República Dominicana } \\
\text { importa del mundo }\end{array}$ & 8560 & 10452 & 23891 & 39391 & 31023 & 262,42 \\
\hline
\end{tabular}




\begin{tabular}{|c|c|c|c|c|c|c|}
\hline $\begin{array}{l}\text { Participación de } \\
\text { Colombia en el } \\
\text { mercado destino }\end{array}$ & 97,91 & 80,23 & 34,74 & 39,57 & 52,00 & $-46,89$ \\
\hline $\begin{array}{l}\text { Participación del } \\
\text { mercado destino en } \\
\text { las exportaciones de } \\
\text { Colombia }\end{array}$ & 2,67 & 7,54 & 28,72 & 20,62 & 16,42 & 514,33 \\
\hline \multirow{5}{*}{$\begin{array}{l}\text { Principales } \\
\text { proveedores de aceite } \\
\text { de palma en el mercado } \\
\text { República Dominicana } \\
\text { (2012) }\end{array}$} & \multicolumn{3}{|c|}{$\begin{array}{l}\text { Principales proveedores en el } \\
\text { mercado (2012) }\end{array}$} & \multicolumn{2}{|c|}{$\begin{array}{l}\text { Participación en el } \\
2012\end{array}$} & Variación 2008 - 2012 \\
\hline & \multicolumn{3}{|c|}{ Colombia } & \multicolumn{2}{|c|}{52,00} & 92,49 \\
\hline & \multicolumn{3}{|c|}{ Honduras } & \multicolumn{2}{|c|}{22,03} & - \\
\hline & \multicolumn{3}{|l|}{ Ecuador } & \multicolumn{2}{|c|}{11,61} & - \\
\hline & \multicolumn{3}{|c|}{ Costa Rica } & \multicolumn{2}{|c|}{3,01} & - \\
\hline \multirow{7}{*}{$\begin{array}{l}\text { Importaciones de } \\
\text { aceites vegetales en } \\
\text { el } 2012\end{array}$} & \multicolumn{3}{|c|}{ Aceites vegetales } & \multicolumn{2}{|c|}{$\begin{array}{l}\text { Participación en el } \\
2012\end{array}$} & Variación 2008 - 2012 \\
\hline & \multicolumn{3}{|c|}{ Aceite de soya } & \multicolumn{2}{|c|}{$69,05 \%$} & $15,73 \%$ \\
\hline & \multicolumn{3}{|c|}{ Aceite de palma } & \multicolumn{2}{|c|}{$13,28 \%$} & $285,92 \%$ \\
\hline & \multicolumn{3}{|c|}{ Aceite de oliva } & \multicolumn{2}{|c|}{$3,70 \%$} & $104,08 \%$ \\
\hline & \multicolumn{3}{|c|}{ Aceite de girasol } & \multicolumn{2}{|c|}{$5,49 \%$} & $129,80 \%$ \\
\hline & \multicolumn{3}{|c|}{ Aceite de colza } & \multicolumn{2}{|c|}{$7,72 \%$} & $192010 \%$ \\
\hline & \multicolumn{3}{|c|}{ Aceite de almendra de palma } & \multicolumn{2}{|c|}{$0,76 \%$} & $3418,52 \%$ \\
\hline $\begin{array}{l}\text { Arancel aplicado por } \\
\text { República Dominicana } \\
\text { para el ingreso de } \\
\text { producto }\end{array}$ & \multicolumn{6}{|c|}{$\begin{array}{l}\text { Colombia: }(18,3 \% \%) \\
\text { Honduras: }(13,5 \%) \\
\text { Ecuador: }(18,3 \%) \\
\text { Costa Rica: }(13,05 \%)\end{array}$} \\
\hline
\end{tabular}

Fuente: Elaboración propia.

\section{Mercado de Estados Unidos}

Estados Unidos es un mercado con muy alto volumen de importaciones de aceite de palma, representado en más de mil millones de dólares en el 2012 y con crecimiento de $2 \%$ entre 2008 - 2012.

La participación de Colombia en el mercado norteamericano es muy baja (inferior al 0,5\% en el 2012) y disminuyó entre 2006 - 2012 en 72\%, por lo tanto se evidecia pérdida de participación en el mercado norteamericano para Colombia en aceite de palma.

En el mercado de Estados Unidos se presenta muy fuerte competencia para Colombia por la alta particpación de las potencias mundiales en este producto, como Malasia e Indonesia. El primero tuvo participación de 93,45\% en el 2012 y crecmiento en el periodo 2008 - 2012 superior al comportamiento del mercado, por lo tanto es altamente dominante en el mercado. Ninguno de los principales competidores en el mercado pagan arancel para el ingreso del producto a Estados Unidos.

A pesar del volumen alto en las importaciones de Estados Unidos en aceite de palma, no es el principal aceite oleaginoso importado por el mercado; en primer 
lugar está el aceite de colza con participación aproximada del 35\% en el 2012, frente al $20 \%$ del aceite de palma.

Tabla 9. Desempeño de Colombia en exportaciones de aceite de palma (1511) en el mercado de Estados Unidos - Valores en miles de dólares

\begin{tabular}{|c|c|c|c|c|c|c|}
\hline & 2008 & 2009 & 2010 & 2011 & 2012 & Variación 2009 - 2013 \\
\hline $\begin{array}{l}\text { Colombia exporta hacia } \\
\text { el mundo }\end{array}$ & 320344 & 138624 & 83180 & 191059 & 188985 & $-41,01$ \\
\hline $\begin{array}{l}\text { Estados Unidos } \\
\text { importa de Colombia }\end{array}$ & 17796 & 4880 & 5788 & 6408 & 5039 & $-71,68$ \\
\hline $\begin{array}{l}\text { Estados Unidos } \\
\text { importa del mundo }\end{array}$ & 1032366 & 714401 & 784397 & 1209158 & 1053058 & 2,00 \\
\hline $\begin{array}{l}\text { Participación de } \\
\text { Colombia en el } \\
\text { mercado destino }\end{array}$ & 1,724 & 0,683 & 0,738 & 0,530 & 0,479 & $-72,24$ \\
\hline $\begin{array}{l}\text { Participación del } \\
\text { mercado destino en } \\
\text { las exportaciones de } \\
\text { Colombia }\end{array}$ & 5,56 & 3,52 & 6,96 & 3,35 & 2,67 & $-52,00$ \\
\hline \multirow{5}{*}{$\begin{array}{l}\text { Principales } \\
\text { proveedores de aceite } \\
\text { de palma al mercado de } \\
\text { Estados Unidos (2012) }\end{array}$} & \multicolumn{3}{|c|}{$\begin{array}{l}\text { Principales proveedores en el } \\
\text { mercado (2012) }\end{array}$} & \multicolumn{2}{|c|}{$\begin{array}{l}\text { Participación en el } \\
\qquad 2012\end{array}$} & Variación 2008 - 2012 \\
\hline & \multicolumn{3}{|l|}{ Malasia } & \multicolumn{2}{|c|}{93,45} & 6,60 \\
\hline & \multicolumn{3}{|l|}{ Indonesia } & \multicolumn{2}{|c|}{4,41} & $-35,69$ \\
\hline & \multicolumn{3}{|l|}{ Holanda } & \multicolumn{2}{|c|}{0,56} & 133,12 \\
\hline & \multicolumn{3}{|l|}{ Colombia } & \multicolumn{2}{|c|}{0,48} & $-71,68$ \\
\hline \multirow{7}{*}{$\begin{array}{l}\text { Importaciones de } \\
\text { aceites vegetales en } \\
\text { el } 2012\end{array}$} & \multicolumn{3}{|c|}{ Aceites vegetales } & \multicolumn{2}{|c|}{$\begin{array}{l}\text { Participación en el } \\
2012 \\
\end{array}$} & Variación 2008 - 2012 \\
\hline & \multicolumn{3}{|c|}{ Aceite de colza } & \multicolumn{2}{|c|}{$34,94 \%$} & $36,95 \%$ \\
\hline & \multicolumn{3}{|c|}{ Aceite de almendra de palma } & \multicolumn{2}{|c|}{$20,52 \%$} & $9,05 \%$ \\
\hline & \multicolumn{3}{|c|}{ Aceite de oliva } & \multicolumn{2}{|c|}{$18,52 \%$} & $-6,40 \%$ \\
\hline & \multicolumn{3}{|c|}{ Aceite de palma } & \multicolumn{2}{|c|}{$20,82 \%$} & $6,26 \%$ \\
\hline & \multicolumn{3}{|c|}{ Aceite de girasol } & \multicolumn{2}{|c|}{$3,36 \%$} & $53,70 \%$ \\
\hline & \multicolumn{3}{|c|}{ Aceite de soya } & \multicolumn{2}{|c|}{$1,53 \%$} & $126,17 \%$ \\
\hline $\begin{array}{l}\text { Arancel aplicado por } \\
\text { Estados Unidos para el } \\
\text { ingreso de producto }\end{array}$ & \multicolumn{6}{|c|}{$\begin{array}{l}\text { Malasia: }(0 \%) \\
\text { Indonesia: }(0 \%) \\
\text { Colombia: }(0 \%) \\
\text { Holanda: }(0 \%)\end{array}$} \\
\hline
\end{tabular}

Fuente: Elaboración propia.

\section{Mercado de Alemania}

Alemania es un mercado muy atractivo por el alto volumen relativo de importaciones en aceite de palma que en el 2012 fue superior a 1300 millones de dólares y creció 17,2\% entre 2008 - 2012.

En un mercado con muy alto volumen y en crecimiento para el producto, Colombia perdió participación entre 2008 - 2012 al pasar del 11,49\% al 1,68\%, respectivamente. 
Aunque sigue siendo un mercado significativo para el sector de Colombia, porque representó en el 2012 el 11,79\% de las exportaciones del producto.

Holanda es el principal país proveedor en aceite de palma al mercado alemán; es de resaltar que Holanda es un país importador y exportador del producto, también exportan al mercado Indonesia, Nueva Guinea y Malasia entre los principales participantes. Indonesia y Malasia deben asumir un arancel de 5,8\% para el ingreso del producto al mercado.

El aceite de palma es el principal aceite oleaginoso importado por Alemania, con participación de $44 \%$ entre los principales aceites importados. No se evidencia sustitución del producto en el mercado en el corto plazo.

Tabla 10. Desempeño de Colombia en exportaciones de aceite de palma (1511) en el mercado de Alemania - Valores en miles de dólares

\begin{tabular}{|c|c|c|c|c|c|c|}
\hline & 2008 & 2009 & 2010 & 2011 & 2012 & Variación 2008 - 2012 \\
\hline $\begin{array}{l}\text { Exportaciones totales } \\
\text { de Colombia en aceite } \\
\text { de palma }\end{array}$ & 320344 & 138624 & 83180 & 191059 & 188985 & $-41,01$ \\
\hline $\begin{array}{l}\text { Alemania importa } \\
\text { desde Colombia }\end{array}$ & 130186 & 43141 & 1443 & 36814 & 22286 & $-82,88$ \\
\hline $\begin{array}{l}\text { Alemania importa de } \\
\text { todo el mundo }\end{array}$ & 1133261 & 993856 & 1169592 & 1357518 & 1328139 & 17,20 \\
\hline $\begin{array}{l}\text { Participación de } \\
\text { Colombia en el } \\
\text { mercado destino }\end{array}$ & 11,49 & 4,34 & 0,12 & 2,71 & 1,68 & $-85,38$ \\
\hline $\begin{array}{l}\text { Participación del } \\
\text { mercado destino en } \\
\text { las exportaciones de } \\
\text { Colombia }\end{array}$ & 40,64 & 31,12 & 1,73 & 19,27 & 11,79 & $-70,99$ \\
\hline \multirow{5}{*}{$\begin{array}{l}\text { Principales } \\
\text { proveedores al mercado } \\
\text { de Alemania (2012) }\end{array}$} & \multicolumn{3}{|c|}{$\begin{array}{c}\text { Principales proveedores en el } \\
\text { mercado (2012) }\end{array}$} & \multicolumn{2}{|c|}{$\begin{array}{l}\text { Participación en el } \\
2012\end{array}$} & Variación 2008 - 2012 \\
\hline & \multicolumn{3}{|l|}{ Holanda } & \multicolumn{2}{|c|}{32,14} & 37,77 \\
\hline & \multicolumn{3}{|l|}{ Indonesia } & \multicolumn{2}{|c|}{24,49} & $-30,13$ \\
\hline & \multicolumn{3}{|c|}{ Nueva Guinea } & \multicolumn{2}{|c|}{13,41} & 165,09 \\
\hline & \multicolumn{3}{|l|}{ Malasia } & \multicolumn{2}{|c|}{11,73} & 71,91 \\
\hline \multirow{7}{*}{$\begin{array}{l}\text { Importaciones de } \\
\text { Alemania en aceites } \\
\text { vegetales en el } 2012 \text { de } \\
\text { los principales aceites } \\
\text { oleaginosos }\end{array}$} & \multicolumn{3}{|c|}{ Aceites vegetales } & \multicolumn{2}{|c|}{$\begin{array}{l}\text { Participación en el } \\
\qquad 2012\end{array}$} & Variación 2008 - 2012 \\
\hline & \multicolumn{3}{|c|}{ Aceite de palma } & \multicolumn{2}{|c|}{$44,12 \%$} & $17,20 \%$ \\
\hline & \multicolumn{3}{|c|}{ Aceite de almendra de palma } & \multicolumn{2}{|c|}{$23,42 \%$} & $10,06 \%$ \\
\hline & \multicolumn{3}{|c|}{ Aceite de girasol } & \multicolumn{2}{|c|}{$11,92 \%$} & $-26,41 \%$ \\
\hline & \multicolumn{3}{|c|}{ Aceite de oliva } & \multicolumn{2}{|c|}{$8,21 \%$} & $-12,83 \%$ \\
\hline & \multicolumn{3}{|c|}{ Aceite de colza } & \multicolumn{2}{|c|}{$9,46 \%$} & $-58,44 \%$ \\
\hline & \multicolumn{3}{|c|}{ Aceite de soya } & \multicolumn{2}{|c|}{$2,86 \%$} & $-69,72 \%$ \\
\hline $\begin{array}{l}\text { Arancel aplicado por } \\
\text { Alemania para el } \\
\text { ingreso del producto } \\
\text { aceite de palma }\end{array}$ & \multicolumn{6}{|c|}{$\begin{array}{l}\text { Holanda: } 0 \% \\
\text { Indonesia: } 5,8 \\
\text { Malasia: }(5,8 \%) \\
\text { Nueva Guinea: } 0 \% \\
\text { Colombia: }(0 \%)\end{array}$} \\
\hline
\end{tabular}

Fuente: Elaboración propia. 


\section{Mercado de Panamá}

Panamá es un mercado con volumen de importación relativamente bajo en aceite de palma, en el 2012 fue de 11,8 millones de dólares y creció en 174\% entre 2008 - 2012.

Colombia aumentó las exportaciones en el mercado de Panamá entre 2008 2012 en $142 \%$, ritmo menor al crecimiento del mercado, por lo que la participación disminuyó en $11 \%$ en el mismo periodo, sin embargo, Colombia es el primer país proveedor del mercado en dicho producto, en el que también participan Costa Rica, Honduras y Malasia principalmente. Todos deben pagar el mismo arancel para el ingreso del producto a dicho mercado.

El principal aceite oleaginoso importado por Panamá es el aceite de soya que participó con el $60,5 \%$ en el 2012, frente al 20,81\% del aceite de palma; aunque el crecimiento de las importaciones hechas por el mercado de aceite de palma son superiores a las del aceite de soya, no se presentaría sustitución en el corto plazo por la alta participación.

Tabla 11. Desempeño de Colombia en exportaciones de aceite de palma (1511) en el mercado de Panamá - Valores en miles de dólares

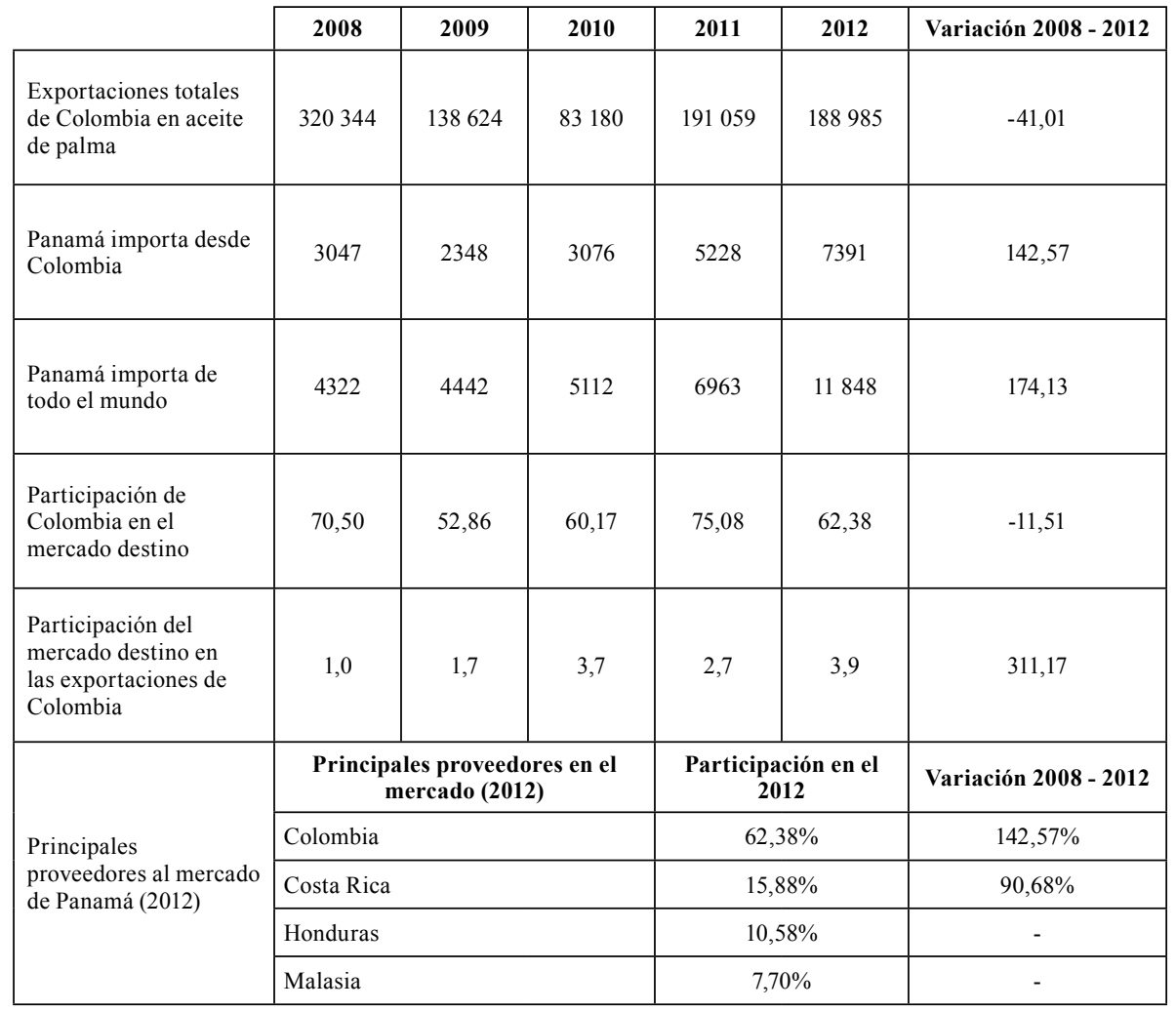




\begin{tabular}{|l|l|c|c|}
\hline \multirow{4}{*}{$\begin{array}{l}\text { Importaciones de } \\
\text { Panamá en aceites } \\
\text { vegetales en el } 2012\end{array}$} & $\begin{array}{c}\text { Participación en el } \\
\mathbf{2 0 1 2}\end{array}$ & Variación 2008 - 2012 \\
\cline { 2 - 4 } & Aceite de soya & $60,52 \%$ & $0,04 \%$ \\
\cline { 2 - 4 } & Aceite de palma & $20,81 \%$ & $174,13 \%$ \\
\cline { 2 - 4 } & Aceite de oliva & $6,67 \%$ & $-33,65 \%$ \\
\cline { 2 - 4 } & Aceite de girasol & $6,65 \%$ & $169,15 \%$ \\
\cline { 2 - 4 } & Aceite de colza & $5,29 \%$ & $1134,43 \%$ \\
\hline $\begin{array}{l}\text { Arancel aplicado por } \\
\text { Panamá para el ingreso } \\
\text { del producto aceite de } \\
\text { palma }\end{array}$ & $\begin{array}{l}\text { Colombia: }(11,1 \%) \\
\text { Malasia: }(11,1 \%)\end{array}$ & $\begin{array}{l}\text { Costa Rica: }(11,1 \%) \\
\text { Honduras: }(11,1 \%)\end{array}$ & \\
\hline
\end{tabular}

Fuente: Elaboración propia.

\section{Balanza comercial relativa sectorial para el aceite de palma de Colombia con los países destino de las exportaciones}

La balanza comercial relativa mide la tendencia importadora o exportadora. Los valores de este indicador están ente -1 y 1 . Cuando el valor se acerca a -1 indica que es un sector donde solo se realizan importaciones. Cuando el valor es 1, o se acerca a 1, significa que es un sector donde solo se exporta. Los valores intermedios negativos reflejan que son mayores las importaciones que las exportaciones, y los valores intermedios positivos muestran que las exportaciones son mayores que las importaciones. Desde un enfoque competitivo, se entiende que tiene más competitividad en la región aquel o aquellos sectores con valores positivos cercanos a 1 (Arias y Segura, 2004).

Se aplica la siguiente fórmula:

$$
B C R_{i j}=\frac{\left(X_{i}^{j}-M_{i}^{j}\right)}{\left(X_{i}^{j}+M_{i}^{j}\right)}
$$

Numerador: Exportaciones de Colombia al país específico de aceite de palma menos importaciones de Colombia del país específico en aceite de palma.

Denominador: Exportaciones totales de Colombia al país específico más importaciones de Colombia provenientes del país específico.

Tabla 12. Balanza comercial relativa de la palma de aceite (1511) en los principales mercados destino de las exportaciones de Colombia

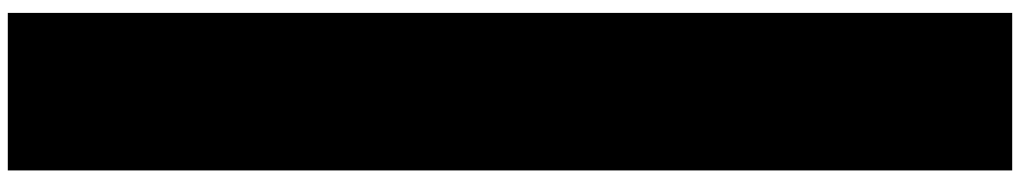




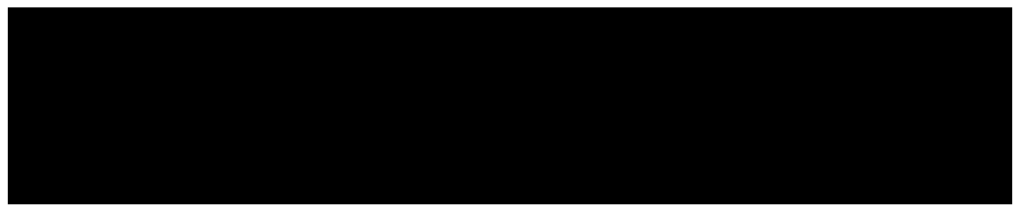

Fuente: Elaboración propia.

La balanza comercial relativa entre todos los países destino de las exportaciones de aceite de palma adquiere el valor de 1, salvo dos excepciones, uno con Brasil en el 2010 y otro con Estados Unidos en el 2011. El valor de este índice refleja la tendencia altamente exportadora de Colombia con los mercados hacia los cuales dirige sus productos. Pero hay que tener en cuenta que Colombia no realiza importaciones, casi en ningún caso, de los países a los cuales exporta. Es por este hecho que el indicador de balanza comercial relativa adquiere alto valor.

Esto refleja que Colombia tiene ventajas en los mercados a los cuales exporta al no presentar amenazas de importaciones provenientes de dichos mercados para el producto aceite de palma.

\section{Índice de ventaja comparativa revelada respecto a un mercado}

Tal como se planteó atrás, establece una relación entre el saldo comercial de una región en un mercado específico para un producto determinado; con el total del intercambio comercial de la región para un producto. Se establece en un tiempo determinado, que generalmente es un año.

$$
I V C R_{i t}^{k}=\frac{\left(X_{i j t}^{k}-M_{i j t}^{k}\right)}{\left(X_{w t i}^{k}+M_{w t i}^{k}\right)}
$$

$X_{i j t}^{k}=$ Exportaciones del producto $\mathrm{k}$ realizadas por el país i (Colombia) al mercado $\mathrm{j}$ en al año t.

$M_{i j t}^{k}=$ Importaciones del producto k realizadas por la región i (Colombia) desde el mercado j en el año t.

$X_{w t i}^{k}=$ Exportaciones del producto $\mathrm{k}$ realizadas por la región (Colombia) i al mundo (w).

$M_{w t i}^{k}=$ Importaciones del producto $\mathrm{k}$ realizadas por la región i (Colombia) de todo el mundo (w).

En el caso específico, se hace el cálculo del Índice de Ventaja Comparativa Revelada para el aceite de palma y aceite de palma de Colombia con respecto los principales mercados de exportación. 
El indicador puede tomar valores positivos o negativos (provenientes del numerador). Un valor positivo expresa una ventaja en los intercambios comerciales derivado del superávit en la balanza comercial sectorial y un valor negativo muestra una desventaja en los intercambios comerciales proveniente de un déficit en la balanza comercial sectorial. Entre mayor sea el valor positivo, es un indicativo de un sector con potencial competitivo; entre mayor sea el valor negativo, indica la carencia de competitividad del sector en el mercado externo.

Tabla 13. Índice de ventaja comparativa revelada para los principales mercados exportadores de aceite de palma de Colombia entre 2008 - 2012

\begin{tabular}{|l|c|c|c|c|c|}
\hline PAÍS & $\mathbf{2 0 0 8}$ & $\mathbf{2 0 0 9}$ & $\mathbf{2 0 1 0}$ & $\mathbf{2 0 1 1}$ & $\mathbf{2 0 1 2}$ \\
\hline Holanda & 0,07 & 0,04 & 0,03 & 0,23 & 0,22 \\
\hline México & 0,004 & 0,17 & 0,14 & 0,02 & 0,10 \\
\hline Brasil & 0,08 & 0,07 & $-0,01$ & 0,14 & 0,10 \\
\hline República Dominicana & 0,02 & 0,04 & 0,04 & 0,04 & 0,05 \\
\hline Chile & 0,02 & 0,02 & 0,03 & 0,02 & 0,02 \\
\hline Panamá & 0,01 & 0,02 & 0,02 & 0,02 & 0,02 \\
\hline Estados Unidos & 0,04 & 0,02 & 0,02 & 0,00 & 0,01 \\
\hline Alemania & 0,38 & 0,20 & 0,00 & 0,04 & 0,01 \\
\hline
\end{tabular}

Fuente: Elaboración propia.

Colombia presenta ventaja comparativa en aceite de palma en todos los mercados a los que realizó exportaciones, ya que en casi todos los casos el valor del índice de ventaja comparativa revelada es positivo, sin embargo, los valores están bastante cercanos a cero, lo que indica la baja participación en cada mercado, esto apoyado en el indicador de balanza comercial relativa que evidencia la ausencia de importaciones realizadas por Colombia en el producto de cada mercado incluido en el análisis. Por tanto, la ventaja comparativa no es muy fuerte en cada mercado. Se destaca ganancia de ventaja en el mercado de Holanda y Brasil en el 2011 y 2012, frente a una pérdida de ventaja en el mercado de Alemania a partir del 2010.

\section{Conclusiones}

Colombia se ubica entre los principales productores y exportadores mundiales de aceite de palma; entre 2008 - 2012 sus exportaciones se han dirigido principalmente a mercados del continente americano, como: Brasil, México, Chile, Panamá, República Dominicana y Estados Unidos, entre otros. Aunque el principal destino del producto entre los años mencionados es Holanda con 37\% en el 2012, al continente europeo se realizaron exportaciones muy bajas. Dentro del periodo analizado las exportaciones colombianas del producto disminuyeron en $41 \%$ frente al crecimiento 
de las importaciones en $403 \%$, lo que evidencia pérdida de competitividad interna del producto y amenaza de sustitución de las importaciones, se destaca el aumento del consumo interno en la producción de biodiésel a partir de aceite de palma que en el 2012 alcanzó 489 mil toneladas.

El comportamiento de los mercados destino de las exportaciones de aceite de palma de Colombia no ha sido homogéneo, mientras que en el 2008 el principal mercado de exportación era Alemania con el 41\%, en el 2012 fue Holanda con 37\%. En el primer país se presentó una variación negativa de $97 \%$ y en el segundo una positiva de $182 \%$. Se ganó además participación en los mercados de México y República Dominicana, entre otros, y se presentó disminución en el mercado de Estados Unidos. Esto evidencia que el producto no ha logrado posiciones competitivas estables en los mercados destino y presenta vulnerabilidad ante comportamientos del mercado.

Colombia presenta ventajas en los mercados de Holanda y México, al tener crecimientos en las exportaciones entre 2008 - 2012, superiores a las importaciones del mercado; en los demás destinos incluidos en el análisis el aceite de palma de Colombia tienen variación menor al comportamiento del mercado. En el mercado de Panamá el producto de Colombia presenta un crecimiento ligeramente inferior al crecimiento del mercado. El aceite de palma de Colombia pierde oportunidades en mercados con dinámica creciente, como: Brasil, República Dominicana, Estados Unidos y Alemania.

Para los principales mercados de exportación de Colombia el aceite de palma es el principal aceite oleaginoso importado en Holanda, México y Alemania; en los demás mercados las importaciones están lideradas por aceites de soya (Panamá, República Dominicana, Chile) de colza (en EE. UU.) y de Oliva (en Brasil).

Existen fuertes competidores como Indonesia y Malasia que son los principales productores y exportadores mundiales; ellos tienen alta participación en los mercados de Holanda, Brasil, Estados Unidos y Alemania, que a su vez son los países con los mayores volúmenes de importación entre los incluidos en el análisis. En los demás mercados se destaca la participación de Ecuador, Honduras y Costa Rica, quienes han ganado presencia internacional y son competidores fuertes para Colombia, porque tienen ventajas comparativas similares representadas en distancia geográfica y en aranceles.

Colombia tiene ventaja arancelaria frente a los competidores en casi todos los mercados a los cuales exporta, el aceite de palma colombiano está libre de arancel en Holanda, Brasil, Chile, Estados Unidos y Alemania. En los demás mercados destino de las exportaciones analizadas debe pagar arancel similar a los principales competidores.

Las principales ventajas comparativas de Colombia para el aceite de palma se presentan en el mercado de Holanda, especialmente para 2011 y 2012; en los mismos años se ganan ventajas en el mercado de Brasil. En los demás países no se evidencia 
aprovechamiento de ventajas comparativas significativas para este producto, pero se presentan oportunidades para el sector palmicultor colombiano, por el volumen de las importaciones, la dinámica del mercado, el posicionamiento del producto frente a los demás aceites oleaginosos y reducción de aranceles.

\section{Referencias}

Arias, J., y Segura, R. (2004). Índice de ventaja comparativa revelada: un indicador del desempeño y de la competitividad productivo-comercial de un país. InterCambio, $I V$. Instituto Interamericano de Cooperación para la Agricultura - IICA.

Artal, A., Castillo, J., \& Requena, F. (2006). Contrastación empírica del modelo de dotaciones factoriales para el comercio interregional de España. Investigaciones Económicas, 30(3), 539-576.

Benítez, L. (2007). El proceso de internacionalización de las empresas en el mundo competitivo y globalizado actual. Contribuciones a la Economía. Revista virtual Eumet. Recuperado de http://www.eumed.net/ce/

Bonifaz, J. L., y Mortimore, M. (1999). Colombia un CANálisis de su competitividad internacional. CEPAL, Santiago de Chile.

Chavarría, H., Sepúlveda, S., \& Rojas, P. (2002). Competitividad de cadenas agroalimentarias y territorios rurales. Elementos conceptuales, No. 1. IICA. Costa Rica.

De Pablo, J., \& Battistuzzi, Á. (2012). Competitividad en el comercio internacional vs ventajas comparativas reveladas caso de análisis: peras. Economia Agrícola, (59), 1, 61-78. São Paulo.

Federación de Cultivadores de Palma - Fedepalma. (2013). Minianuario Estadístico 2013. Principales cifras de la agroindustria de la palma de aceite en Colombia. Bogotá.

Jung, A., Garbarino, P., Jerusalmi, C., Durán, C., \& Plottier, C. (2006). Un aporte a los procesos de construcción competitiva basados en clusters. Cuaderno de Economía, (1), 91-106.

Labarca, N. (2007). Consideraciones teóricas de la competitividad empresarial. Omnia, 13(2).

Lombana, J., \& Rozas, S. (2009). Marco analítico de la competitividad: Fundamentos para el estudio de la competitividad regional. Pensamiento \& Gestión, 26.

Olivera, M., Escobar, D., Rojas, N., Moreno, J., Quintero, C. P., \& Tibocha, A. (2011). Caracterización del empleo en el sector palmicultor colombiano. Cuadernos de Fedesarrollo. Bogotá. 
Porter, M. E. (1999). Ser competitivo: Fronteras en expansión. Harvard Deusto business review, (91), 34-36.

Porter, M. E., \& Kramer, M. R. (2006). Estrategia y sociedad. Harvard Business Review, 84(12), 42-56.

Villazul, J. (2004). Trayectoria tecnológica y ciclo de vida de las empresas: una interpretación metodológica acerca del rumbo de la innovación. Contaduría y Administración, 214,83-96. Universidad Nacional Autónoma de México.

\section{Referencias Web}

Trademap. Base de datos estadísticas del comercio para el desarrollo internacional de las empresas. Dirección electrónica: http://www.trademap.org/Index.aspx 